\title{
Load Balancing in Network to Increasing Performance Ratio of Packet Delivery using Ant Colony Optimization
}

\author{
Niitn Girme \\ (Student) \\ Dr. D.Y.Patil College of Engineering, Ambi
}

\begin{abstract}
Now a day's networks grow to become more and more complicated. Network can be wireless or wired with number of nodes. The problem remains the same in order to get best performance of packet delivery. There is need to find shortest path in order accomplish the delivery of packet. It's very difficult task to manage the load balance in between traffic and routing packets to the destination. Those nodes carrying excess data can become congested in network and chance to data lost. The nature gives the solution to managing the load balancing in between network to handle largest influx of traffic. The ants goes outside for finding their foods and come to their nest. The ants move across the network in between randomly chosen pairs of nodes; as they move they deposit simulated pheromones as a function of their distance from their own source node, and the congestion encountered on their root. They select their path at each intermediate node according the distribution of simulated pheromones at each node. For each intermediate node the calls come from source to reach destination as a function of pheromone distributation at each intermediate node. The performance of the network is measured by load balancing in network, performance ratio, and packet loss ratio as compared to open shortest path first algorithm.
\end{abstract}

\section{General Terms}

Load balancing, Ant colony optimization, AntNet optimization, Ant colonies.

\section{Keywords}

Influx of traffic, pheromone table

\section{INTRODUCTION}

Load balancing is a technique to spread work between two or more computers/networks, other resources in order to get optimal maximize throughput, minimize response time and resource utilization in network. Load balancing can be useful when dealing with redundant communications (networks) links. Load balancing is basically the construction of call routing schemes which successfully distribute the changing load over the networks and minimize lost calls. Absolutely it is possible to determine the shortest routes from every node to every other node in the network. In this way the average utilization of nodes will be minimized, but it is not necessarily the ideal way to avoid congestion of node, as this has to do with how the traffic on the network is distributed. This type distributed system controlling by means of a single central controller has several disadvantages. The controller usually needs to have current knowledge about the entire system, necessitating communication links of system from every part of the system to controller. Since technology growing faster, people are more interested to finding ways that connect each other faster than last one. However many technologies invented for this problem, some that are highly effective in individual cases. Ants first evolved 120 million years ago, they found more than 10,000 different species. They leaved in different colonies. For moving from one place to other ants having ability to create their own path called as "ant streets". Streets are in the form of long, bidirectional lanes of single pathways in which they navigate landscapes in order to reach a destination in optimal time. Ants drop the pheromone on the ant streets which guide them using a shortest path mechanism. This technique allows finding optimal minimum path. In the year 1990's Computer Scientist researched new routing algorithm in result Ant Colony Optimization, it minimize number of nodes that are useful to connect in order to complete network and minimizing load and increasing reliability. Marco Dorigo and Thomas Stutzle gave the design to implement ANTNet, it conclude how the algorithm perform and how it could be further implement [16].

\section{LITERATURE, SURVEY}

\subsection{The Ants}

Ant as a single individual has a very limited effectiveness. But as a part of a well-organized colony, it becomes one powerful agent, working for the development of the colony. The ant lives for the colony and exists only as a part of it. Ant colonies are sometimes described as superorganism because it appears to operate as a unified entity. Each ant is able to communicate, learn, cooperate, and all together they are capable of develop themselves and colonies a large area. They manage such great successes by increasing the number of individuals and being exceptionally well organized. The self organizing principles they are using allow a highly coordinated behavior of the colony, furthermore bring them to accomplish complex tasks, whose difficulty far exceed the individual capabilities of a single ant. Ants communicate to one another by laying down pheromones along their trails, so where ants go within and around their ant colony is a stigmergic system. Similar phenomena can be observed for some animals, such as termites, which use pheromones to build their very complex nests by following a simple decentralized rule set. In many ant species, ants walking from or to a food source, deposit on the ground a substance called pheromone. Other ants are able to smell this pheromone, and its presence influences the choice of their path, that is, they tend to follow strong pheromone concentrations. The pheromone deposited on the ground forms a pheromone trail, which allows the ants to find good sources of food that have been previously identified by other ants[16].

\subsection{Ants and Pheromones}

The ants begin by walking randomly. They cannot see the ground and have a very limited view of what is around them. Therefore, if the ground has not been explored yet, they will just wander and take random decision at each crossroads. After a while, the places around the nest will be all explored. The ants will get to know that by the marking done by the previous ants. Indeed, they will leave behind them the famous pheromones and inform the other ants that the way is already explored. 


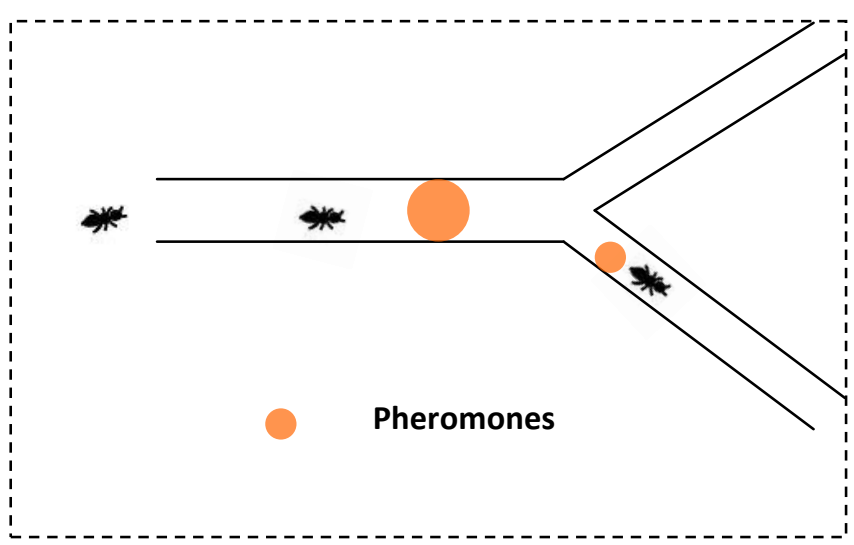

Fig. 1: Ants have ability to take decision

The concentration of pheromones depends on the number of ants who took the way, the more ants taking the way, the more pheromones. The configuration is as shown in figure 1.2: the nest of a colony of ants is connected to the food via two bridges of the same length. In such a setting, ants start to explore the surroundings of the nest and eventually reach the food source. Along their path between food source and nest, ants deposit pheromones. Initially, each ant randomly chooses one of the two bridges. However, due to random fluctuations, after some time one of the two bridges presents a higher concentration of pheromones than the other and, therefore, attracts more ants. This brings a further amount of pheromone on that bridge making it more attractive with the result that after some time the whole colony converges toward the use of the same bridge.

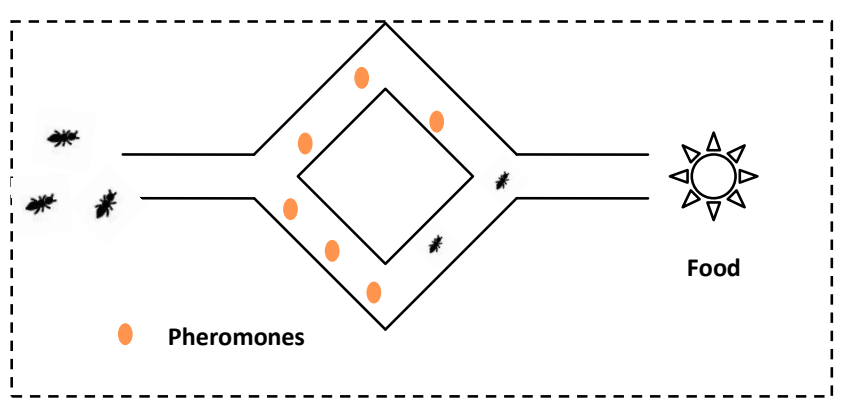

Fig. 2: Ants exploring the double bridge

The ants taking the shorter path will reach the food source before the others, and leave behind them the trail of pheromones. After reaching the food, they will turn back and try to find the nest. At the cross, one of the paths will contain pheromones although the other one will be not explored. Hence the ant which carries the food will take the path already explored, as it means it is the way to the nest. As the ant is choosing the shortest way and will continue to deposit pheromones, the path will therefore become more attractive for others. The ants who took the long way will have more probability to come back using the shortest way, and after some time, they will all converge toward using it. Consequently, the ants will find the shortest path by themselves, without having a global view of the ground. By taking decision at each cross according to the pheromones amount, they will manage to explore, find the food, and bring it back to the nest, in an optimized way.

\section{PROBLEM FORMULATION}

In telecommunication network, the calls between two nodes are routed through number of intermediate hopes. Load balancing it is the process of constructing calls in network and distribute data in network without loss. Ant Based Control to managing load balance in network is suitable. Following are the parameters used to implement artificial ant in network

\subsection{Pheromone Table Update Criteria}

In a computer-based simulation, the ants are replaced by agents who will explore the ground; let pheromones and once the goal reached try to come back. Goss et al., developed a model to explain the behavior observed in the double bridge experiment. Assuming that after $t$ time units since the start of the experiment, $\mathrm{m} 1$ ants had used the first bridge and $\mathrm{m} 2$ the second one, the probability $\mathrm{p} 1$ for the $(\mathrm{m}+1)$ th ant to choose the first bridge can be given by:

$$
p_{1(m+1)}=\frac{\left(m_{1}+k\right)^{h}}{\left(m_{1}+k\right)^{h}+\left(m_{2}+k\right)^{h}}
$$

where parameters $\mathrm{k}$ and $\mathrm{h}$ are needed to fit the model to the experimental data. The probability that the same $(m+1)$ th ant chooses the second bridge is $\mathrm{p} 2(\mathrm{~m}+1)=1-\mathrm{p} 1(\mathrm{~m}+1)$. Monte Carlo simulations, run to test whether the model corresponds to the real data, showed very good fit for $\mathrm{k}=20$ and $\mathrm{h}=2$.

\subsection{How Calls are Route in Network?}

To determine look for a call artificial ants look in pheromone table before moving from source node to destination. Routes are valid if destination is clear to reach the ant. If route is congested then newly arriving call pressure the load on exiting node, it means open the delay mechanism. The relationship between calls, nodes, pheromone table and ants as follows.

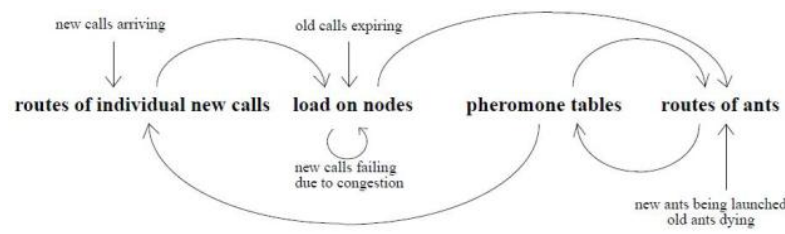

Fig. 3: Relationship between calls, node utilization, pheromone tables and ants.

\section{METHODOLOGY}

\subsection{AntNet Algorithm}

The AntNet first version, presented in 1997 [Dorigo et al 97], will be denominated AntNet1.0, and the second version published in 1998 [Dorigo et al 1998] will be called AntNet2.0

Suppose a data network, with $\mathrm{N}$ nodes, where $\mathrm{s}$ denotes a generic source node, when it generates an agent or ant toward a destination d. Two types of ants are defined:

1. Forward Ant, denoted $F_{s \rightarrow d}$, which will travel from the source node $S$ to a destination $d$.

2. Backward Ant, denoted $B_{s \rightarrow d}$ that will be generated by a forward ant $F_{s \rightarrow d}$ in the destination d, and it will come back to $\mathrm{s}$ following the same path traversed by $F_{s \rightarrow d}$, with the purpose of using the information already picked up by $F_{s \rightarrow d}$ in order to update routing tables of the visited nodes

Every ant transports a stack $S_{s \rightarrow d}(k)$ of data, where the k index refers to the ke-st visited node, in a journey, where $S_{s \rightarrow d}(0)=\mathrm{s}$ and $S_{s \rightarrow d}(m)=\mathrm{d}$, being $\mathrm{m}$ the amount $\mathrm{o}$ 
jumps performed by $F_{s \rightarrow d}$ for arriving to d. Let $\square$ be any network node; its routing table will have $\mathrm{N}$ entries, one for each possible destination. Let $\mathrm{j}$ be one entry of $\square$ routing table (a possible destination). Let $N_{k}$ be set of neighboring nodes of node $\square$. Let $P_{j i}$ be the probability with which an ant or data packet in $\square$ jumps to a node i, i $\in N_{k}$ when the destination is $\mathrm{j}(j \neq k)$. Then, for each of the $\mathrm{N}$ entries in the node k routing table, it will be $N_{k}$ values of $P_{j i}$ subject to the condition:

$$
\sum_{i \in N k} P_{i j}=1 ; j=1, \ldots, N
$$

The following lines show AntNet pseudo code, using the symbols and nomenclature already presented:

\section{BEGIN}

\{Routing Tables Set-Up: For each node k the routing tables are initialized with a uniform distribution:

$$
P_{i j}=\frac{1}{N_{k}}, \forall i \in N_{k}
$$

DO always (in parallel)

\section{\{}

STEP 1: In regular time intervals, each node s launches an $F_{S \rightarrow d}$ ant to a

randomly chosen destination $\mathrm{d}$.

/*During its trip to d, when $F_{S \rightarrow d}$ reach a node $\mathrm{k},(\mathrm{k} \neq \mathrm{d})$, it does step $2 \%$

DO (in parallel, for each $F_{s \rightarrow d}$

\section{\{}

STEP 2: $F_{s \rightarrow d}$ pushes in its stack $S_{S \rightarrow d}(k)$ the node identifier and the time elapsed between its launching from $s$ to its arriving to $\square$.

$F_{S \rightarrow d}$ selects the next node to visit in two possible ways:

(a) It draws between i nodes, $\mathrm{i} \rightarrow N_{k}$, where each node $\mathrm{i}$ has a $P_{d i}$ probability (in the $\square$ routing table) to be selected.

IF the node selected in (a) was already visited

(b) It draws again, but with the same probability for all neighbor nodes $\mathrm{i}, \mathrm{i} \rightarrow N_{k} . \mathrm{Fs} \rightarrow \mathrm{d}$ jumps to chosen node.

IF the selected node was already visited

STEP 3: A cycle is detected and $F_{S \rightarrow d}$ pops from its stack all data related to the cycle nodes, since the optimal path must not have any cycle. $F_{s \rightarrow d}$ comes back to step 2 (a).

END IF

END IF

\section{\} WHILE jumping node $\neq \mathrm{d}$}

STEP 4: $F_{S \rightarrow d}$ generates another ant, called backward ant $B_{s \rightarrow d} . F_{s \rightarrow d}$ transfers to
$B_{S \rightarrow d}$ its stack $S_{S \rightarrow d}$ and then dies.

$/ * B_{s \rightarrow d}$, will follow the same path used by $F_{S \rightarrow d}$, but in the opposing

direction, that is, from $\mathrm{d}$ to $\mathrm{s} * /$

DO (in parallel, for each $B_{S \rightarrow d}$ ant)

\{

/*When $B_{s \rightarrow d}$ arrives from a node $\mathrm{f}, \mathrm{f} \in N_{k}$ to a $\square$, it does step 5*/

STEP 5: $B_{s \rightarrow d}$ updates the $\square$ routing table and list of trips, for the entries regarding to nodes $\square$, between $\square$ and $\mathrm{d}$ inclusive, according to the data carried in $S_{s \rightarrow d}\left(k^{\prime}\right)$.

IF $\square \neq \mathrm{s}$

$B_{s \rightarrow d}$ will jump from k to a node with identifier given by $S_{s \rightarrow d}(k-1)$

END IF

\} WHILE $(\square \neq \mathrm{s})$

\}

\}

END

END

The routing table and list of trips updating methods for $\mathrm{k}$ are described as follows:

1. The $\mathrm{k}$ routing table is updated for the entries corresponding to the nodes $\square$ ' between $\square$ and d inclusive. For example, the updating approach for the d node, when $B_{s \rightarrow d}$ arrives to $\square$, coming from $\mathrm{f}$, $\mathrm{f} \in N_{k}$ is explained, next:

A $P_{d f}$ probability associated with the node $\mathrm{f}$ when it wants to update the data corresponding to the $\mathrm{d}$ node is increased, according to:

$$
P_{d f} \leftarrow P_{d f}+(1-\tilde{\Gamma}) \cdot\left(1-P_{d f}\right)
$$

where $r^{\prime}$ is an dimensional measure, indicating how good (small) is the elapsed trip time $\mathrm{T}$ with regard to what has been

observed on average until that instant. Experimentally, r' is expressed as:

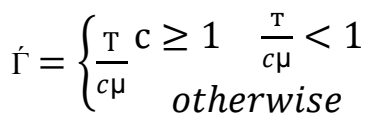

1

where: $\mu$ is the arithmetic observed trip time T average. $\mathrm{c}$ is a scale factor experimentally chosen like 2 (Dorigo 1997). More details about $\mathrm{r}^{\prime}$ and its significance can be found in (Dorigo 1997).

The other neighboring nodes $(j \neq f) P_{d f}$ probabilities associated with node $\square$ are diminished, in order to satisfy equation (1), through the expression: 


$$
P_{d f} \leftarrow P_{d f}+(1-\hat{\Gamma}) \cdot P_{d f} \quad \forall j \in N k, j \neq f
$$

A list $\operatorname{trip}_{k}$ ? $2 i \sigma i^{2}$, ? $\mathrm{i}, \sigma i^{2}$ of estimate arithmetic mean values $? i$ and associated variances $\sigma i^{2}$ for trip times

from node $\mathrm{k}$ to all nodes $\mathrm{i}(\mathrm{i} \neq \mathrm{k})$ is also updated. This data structure represents a memory of the network state as seen by node $\mathrm{k}$. The list trip is updated with information carried by $B_{s \rightarrow d}$ ants in their stack $S_{s \rightarrow d}$. For any node pair sourcedestination, $\mu$ after $(n+1)$ samples $(n>0)$ is calculated as follows:

$$
?_{n-1}=\frac{n ?_{x}+x_{n}+1}{n+1}
$$

where: $\mathrm{xn}+1$ trip time $\mathrm{T}$ sample $\mathrm{n}+1, \mu \mathrm{n}$ arithmetic mean after $\mathrm{n}$ trip time samples [16].

\section{GRAPHICAL RESULTS}

The networks become nowadays more and more complicated, with moving nodes, varying loads, etc. The users however expect more quality and more services despite the growing complexity of the networks. The theses which will be analyzed in the following study some adaptations of the Ant Colony Optimization to routing protocols, and often compare its efficacy to the current routing algorithms. Most of the papers see in the ACO a great tool for wireless Ad Hoc networks as it has a strong capacity to adapt to changes. However, some new algorithms based on ACO are also analyzed for wired networks and are giving encouraging results.

The comparison between ACO and traditional routing algorithms is done with analyzing:

- The performance ratio of packet delivery

- The energy consumption by nodes

- The packet delaying ratio

\subsection{Performance Ratio of Packet Delivery}

In below graph, ACO in red color gives good performance as compare to OSPF by managing load balancing in the network. It shows the performance in percentage (\%). For 20 nodes ACO gives $70 \%$ performance and OSPF gives $50 \%$ performance. See Fig, 4.

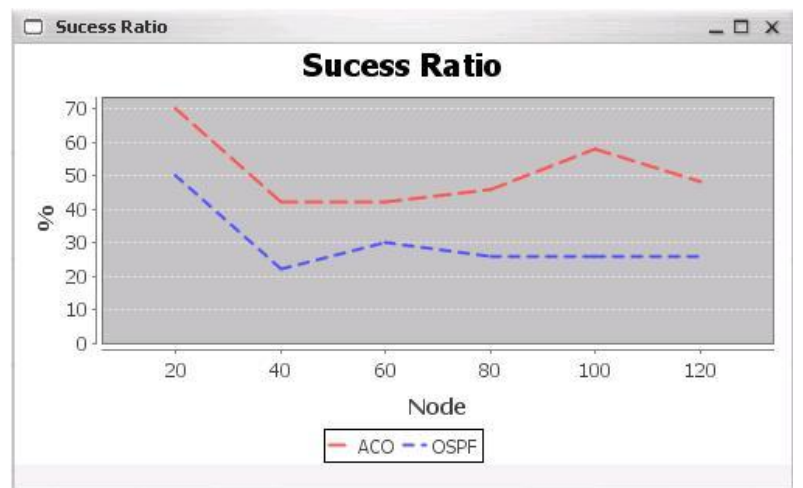

Fig 4: Success ratio between ACO and OSPF

\subsection{Energy Consumption by Nodes}

For delivering a packet to the destination from source some energy is required. ACO consume less energy than OSPF as shown in below graph (red dotted line). OSPF take more energy to deliver packets to the destination. See Fig, 5.

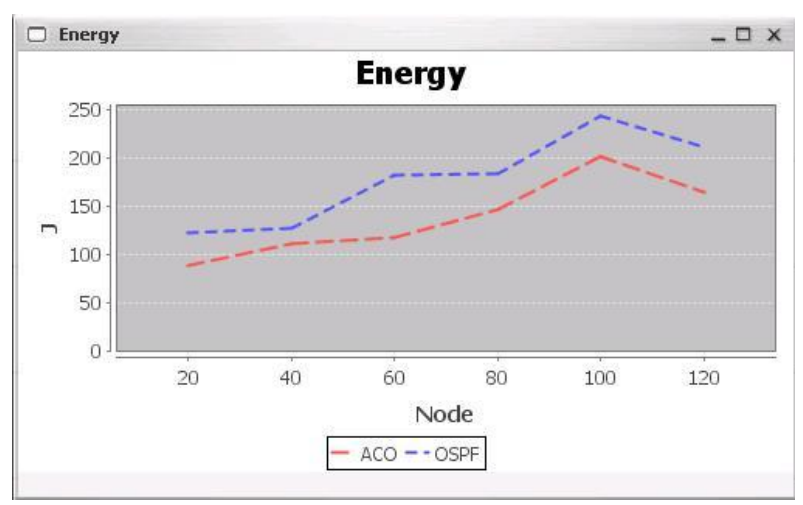

Fig 5: Energy consumption between ACO and OSPF

\subsection{Packet Delaying Ratio}

Due to network congestion and overhead OSPF delaying to deliver the packet to the destination, for delivering 100 packets to destination OSPF take 250 mili-seconds (MS). In other hand ACO take only 200 MS to deliver the packets to the destination. See Fig, 6 .

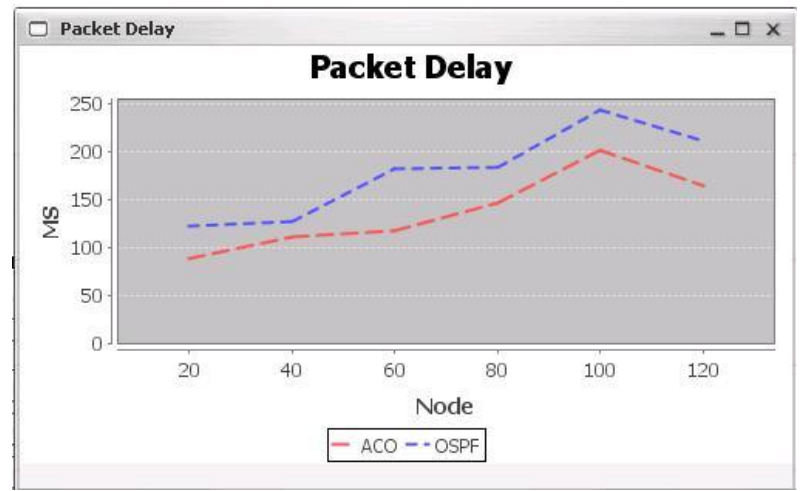

Fig 6: Packet Delaying between ACO and OSPF

\section{CONCLUSION}

As we know the current telecommunication networks suffer from network issues such as network congestion which causes failing of calls in network. For that purpose required routing algorithm as well load sharing algorithm to minimize the effect of congestion. This Java Simulation show that how loads (large traffic as well as large packets) in the network should be managed to get optimal resource utilization, maximize throughput, and minimize response time with comparing Ant Colony Optimization Algorithm and Open Shortest Path First (OSPF). As a result I found that Any Colony Optimization (ACO) is better method than OSPF for balancing the load in network to increasing performance ratio of packet delivery in the networks.

\section{REFERENCES}

[1] B. Barán, M Almirón, E. Chaparro. 'Ant Distributed System for Solving the Traveling Salesman Problem'. pp. 779-789. Vol. 215 th Informatic Latinoamerican Conference-CLEI,. Paraguay (1999). 
[2] B. Barán, M Almirón, E. Chaparro. 'Ant Distributed System for Solving the Traveling Salesman Problem'. pp. 779-789. Vol. 215 th Informatic Latinoamerican Conference-CLEI,. Paraguay (1999).

[3] M. Dorigo, V. Maniezzo, A. Colorni. 'The Ant System: Optimization by a colony of cooperating agents'. pp. 113. Vol. 26-Part B. IEEE Transactions on Systems, Man, and Cybernetics,. Number 1 (1996).

[4] M. Dorigo, G. Di Caro. 'AntNet. Distributed Stigmergetic Control for Communications Networks'. pp. 317-365 Journal of Artificial Intelligence Research. Number 9 (1998).

[5] [Schoonderwoerd et al 97] R. Schoonderwoerd, O. Holland, J. Bruten. 'Ant-like agents for load balancing in telecommunications networks'. Hewlett-Packard Laboratories, Bristol-England (1997).

[6] Beckers, R., Holland, O.E., \& Deneubourg, J.L. (1994). From local actions to global tasks: Stigmergy and Collective Robotics. In R.A. Brooks, \& P. Maes (Eds.) Artificial Life IV, Cambridge, MIT Press. p. 181-189.

[7] Beckers, R., Deneubourg, J.L., \& Goss, S. (1992).Trails and U-turns in the Selection of a Path by the Ant Lasius Niger. In J. theor. Biol. 159, 397-415.

[8] Stickland, T.R., Tofts, C.M.N., \& Franks, N.R. (1992). A path choice algorithm for ants. In Naturwissenschaften $79,567-572$.

[9] Goss. S., Beckers, R., Deneubourg, J.L., Aron, S., \& Pasteels, J.M. (1990). How trail-laying and trail following can solve foraging problems for ant colonies. In R.N. Hughes (Ed.) NATO AS1 Series, Vol. G20 Behavioral mechanisms of food selection, Springer Verlag.

[10] Deneubourg, J.L., Goss, S., Franks, N., Sendova-Franks, A., Detrain C., \& Chrétien, L. (1990). The dynamics of collective sorting robot-like ants and ant-like robots. In J.-A. Meyer \& S. Wilson (Eds.), From Animals to Animats: Proceedings of the first international conference on simulation of adaptive behavior. Cambridge,MITPress.

[11] Beckers, Deneubourg \& Goss, 1992; Deneubourg \& Goss, 1989; Goss et.al., 1990; Franks, 1989.

[12] Beckers, Holland \& Deneubourg, 1994; Russell 1995; Deveza et.al., 1994.

[13] A. Shankar, C. Alaettinoglu, I. Matta. 'Performance Comparison of Routing Protocols using MaRS. Distance Vector versus Link-State'. Technical Report, MarylandUSA (1992)

[14] Modeling the collective building of complex architectures in social insects with lattice swarms,
Theraulaz and Bonabeau, Journal of Theoretical Biology, 1995.

[15] Bert Hölldobler, E.O. Wilson: "Journey to the Ants: A Story of Scientific Exploration, 1994, ISBN 0-67448525-4.

[16] http://ethesis.nitrkl.ac.in/1128/1/thesis.pdf

[17] Grid Load Balancing Using Ant Colony Optimization Nasir, H.J.A. ; Ku-Mahamud, K.R. Computer and Network Technology (ICCNT), $2010 \quad$ Second International Conference onDigital Object Identifier: 10.1109/ICCNT.2010.10 Publication Year: 2010 , Page(s): $207-211$

[18] Load Balancing of Nodes in Cloud Using Ant Colony Optimization Nishant, K. ; Sharma, P. ; Krishna, V. ; Gupta, C. ; Singh, K.P. ; Nitin, N. ; Rastogi, R.Computer Modelling and Simulation (UKSim), 2012 UKSim 14th International Conference on Digital Object Identifier: 10.1109/UKSim.2012.11

[19] Load Balancing in Non-dedicated Grids Using Ant Colony Optimization Yixiong Chen Semantics, Knowledge and Grid, 2008. SKG '08. Fourth International Conference onDigital Object Identifier: 10.1109/SKG.2008.41

[20] A Survey of Ant Colony Optimization-Based Approaches to Routing in Computer Networks Janacik, P. ; Orfanus, D. ; Wilke, A.Intelligent Systems Modelling \& Simulation (ISMS), 2013 4th International Conference on Digital Object Identifier: 10.1109/ISMS.2013.20 Publication Year: 2013 , Page(s): $427-432$

[21] Multiple Ant Colony Routing Optimization Based on Cloud Model for WSN with Long-Chain Structure Yongli Zhu ; Junyan Zhang ; Lifen Li ; Wei Peng Wireless Communications Networking and Mobile Computing (WiCOM), 2010 6th International Conference on Digital Object Identifier: 10.1109/WICOM.2010.5601101Publication Year: 2010 , Page(s): $1 \quad-4$

[22] http://en.wikipedia.org/wiki/Ant_colony_optimization_al gorithms

[23] http://www.ijarcsse.com/docs/papers/Volume_4/6_June2 014/V4I6-0186.pdf

[24] ftp://labattmot.ele.ita.br/ele/jrsantos/Leitura/ACO/ANTrapport.pdf

[25] http://ethesis.nitrkl.ac.in/1321/1/project.pdf

[26] http://research.ijcaonline.org/volume39/number5/pxc387 7068.pdf

[27] http://enggjournals.com/ijcse/doc/IJCSE10-02-02-05.pdf 